\title{
Influence of visual guidance on braille recognition: Low lighting also helps touch
}

\author{
MORTON A. HELLER \\ Winston-Salem State University, Winston-Salem, North Carolina
}

\begin{abstract}
This study was an attempt to clarify the mechanisms responsible for the benefits of visual guidance in tactual braille recognition. Subjects touched $+90^{\circ}$ tilted braille under normal room lighting, or with low lighting, with or without visual guidance. Both visual information about finger angle and spatial reference information were manipulated with stained glass and lightemitting diodes. The provision of visual information about finger angle alone was no help to braille recognition, and performance was low. Adding visual spatial reference information to vision of finger angle raised performance. However, recognition accuracy was also substantially improved by low lighting. The benefits of darkness for haptics did not generalize to the reading of upright, two-letter braille words. It was proposed that extraneous visual information may distract sighted subjects in haptic tasks that require mental rotation of visual images.
\end{abstract}

Providing concurrent information from more than one sense can aid the accuracy of perceptual judgments (see Warren \& Rossano, 1991). Visual guidance (VG) of touch leads to improved relative smoothness judgments of textured surfaces (Heller, 1982). Warren and Rossano suggested that vision of hand motions provides more precise control of the rate of tactual exploration. In addition, VG of touch can aid subjects as they attempt tactual identification of braille (Heller, 1985, 1987, 1989b). Visual regulation of hand movements is likely to help a sighted person track lines of braille. Thus, subjects are far less likely to stray from lines of braille when vision is available to guide their tactual exploration. The effect of visual guidance also extends to pattern identification (see Heller, 1989b). Subjects have shown low performance (43\% correct) when attempting visual matches to $+90^{\circ}$ tilted braille under "normal" control conditions, with the braille flat on a horizontal shelf (Heller, 1989b, 1992a). Visually guided touch improves performance greatly (to $66 \%$ correct for $+90^{\circ}$ tilted braille; Heller, 1989b).

How do we explain the benefits of VG? One possibility is that VG allows the generation of visual images. Thus, plastic stained glass was used in prior research to allow sight of the hand touching a surface, but also to eliminate vision of surface textures or the braille itself (Heller, $1982,1983,1985)$. The visual imagery explanation was suggested by an interesting finding. Naive sighted subjects can identify braille by watching other subjects touch

Preparation of this report and some of the research were supported by NIH MBRS Grant 2 SO6 RR-08040. Faith Heller provided helpful comments on an earlier version of the manuscript. I wish to thank Tamala Joyner. Aretha Jones, and Connie Crawford for assistance with data collection and data analyses. The results were presented at the annual meeting of the North Carolina Cognition Group, Duke University, Feb. 6, 1993. Send reprint requests and inquiries to M. A. Heller, Psychology, Winston-Salem State University, Winston-Salem, NC 27110. an invisible, embossed surface (Heller, 1985). Thus, naive subjects may try to trace braille patterns, and they will engage in vertical motions when tracing the braille character representing the letter B (two vertical dots). These scanning motions are characteristic of the novice or poor braille reader, but are not obvious in skilled readers. Sight of these scanning motions could permit the generation of visual representations. Subjects presumably could translate the sight of kinesthetic patterns into visual images. Many researchers have suggested that visual recoding of tactual information can aid haptic perception in sighted people (Attneave \& Benson, 1969; McKinney, 1964; Pick, 1974; Revesz, 1950).

The results of a recent experiment, however, may force us to reject the imagery explanation of the visual guidance effect. VG aids braille recognition for both upright and tilted braille. However, Heller (1989b, Experiment 5) had subjects touch $+90^{\circ}$ tilted braille in the dark while they viewed a single light-emitting diode (LED) on the upper surface of their fingernails. The stained glass allowed sight of scanning motions, but visual spatial reference cues were eliminated. The subjects were unable to see the angle of their fingers in relation to the lines of braille. Under these circumstances, performance was similar to that found without VG (34\% correct). This means that the visual imagery explanation cannot explain the benefits of VG without spatial reference information. Heller suggested that VG might aid tactual exploration by providing a spatial frame of reference. This consists of information about the horizontal or vertical, within which to interpret tactual information (Millar, 1981, 1991).

Recently, Millar (1991) described a categorization scheme for haptic shape tasks and provided a detailed discussion of the importance of spatial reference information for touch. Shape coding is a special problem for braille, since the patterns are small. Furthermore, braille characters lack redundancy, since they are derived from 
a six-dot matrix. Braille patterns have few internal features that could serve as spatial anchor points. Millar has suggested that higher order, as well as low-order, cognitive skills are involved in braille reading, which is very difficult for the novice. Observation of subject behavior shows that a variety of clumsy, "scrubbing" behaviors are frequent in new braille readers. Skilled readers rarely engage in "scrubbing" and show smooth, lateral scanning of lines of braille (also see Foulke, 1991).

The present research was designed to gather further information about the precise nature of the visual information that was responsible for the previously reported VG effect on braille recognition. As in prior research, darkness, LEDs, and stained glass were used to manipulate visual information about tactual exploration. Control subjects examined braille without VG, in normal room lighting or in relative darkness (Heller, 1989b, 1992a).

\section{EXPERIMENT 1 \\ The Effect of Visual Guidance on $+90^{\circ}$ Tilted Braille}

This experiment was designed to determine the nature of the benefits of VG on tactual exploration. VG might provide assistance to touch via sight of scanning movements (Heller, 1985) or through the provision of a visual frame of reference. This hypothesis was suggested by the damaging effects of finger tilt on braille recognition (Heller, 1987). Sighted individuals made visual matches to embossed braille characters that were tilted $+90^{\circ}$ from the upright. Some observers were permitted VG of tactual exploration; control subjects were denied visual regulation. VG was accomplished by subjects' viewing their tactual exploration through plastic stained glass. In addition, low lighting eliminated sight of the finger, hand, embossing, and surfaces.

One control group of subjects was tested in relative darkness, but without VG. These dark-adapted subjects had a small "submini" lamp above the visual matching code. A second group was permitted VG, which consisted of vision of finger angle. These subjects had two small LEDs attached to the upper surface of the finger (see Heller, 1989b). Another group was given visual information about both the angle of the exploratory finger (via two LEDs) and the angle of the lines of braille-that is, visual spatial reference information. These subjects also had a line of LEDs above each line of braille. A control group examined the braille with normal room lighting.

The low-light control group was included to determine whether irrelevant visual stimulation from the experimental environment could interfere with the subjects' mental rotation of "visual" representations of haptic input. This low-light condition was suggested by observation of the behavior of some individuals in "normal" bimodal experimental conditions in past research. Some subjects seemed to avert their gaze as they touched textures or patterns in prior research. A subject in an ongoing map study reported that closing her eyes, even while she was blind- folded, helped her generate visual images of the tactile maps. In addition, educators of the visually impaired have been known to blindfold people with low vision to facilitate instruction in mobility skills (Lauren, 1988).

\section{Method}

Subjects. There were 48 sighted subjects -28 females and 20 males-recruited from an undergraduate population. All observers were unfamiliar with braille.

Stimuli and Apparatus. The stimuli were standard braille characters representing the letters B-J. The patterns were embossed on plastic cards obtained from the American Foundation for the Blind. Standard braille uses a $3 \times 2$ matrix of dots and is about $6 \mathrm{~mm}$ high; the letters B-J are only about $4 \mathrm{~mm}$ high. The braille patterns were all tilted $+90^{\circ}$ from the upright. The stimuli were arranged randomly in five horizontal rows, with five characters in each row. A final row contained two braille patterns. The beginning and end of each line of braille were marked by thumbtacks pushed into the braille paper. For VG subjects, a box covered with stained glass was used to permit sight of scanning movements and the LEDS, while it eliminated vision of the dots and the subjects' hands (see Heller, 1982, 1983, 1985, 1989b, for further details). In normal room lighting, the stained glass allows the observer blurry vision of his/her finger, but renders embossing and other material invisible. However, in the VG conditions of the present experiment, lighting was reduced and overall room lighting was limited to light from three submini lamps $(1.5 \mathrm{~V}, 25 \mathrm{~mA} ; 0.81 \mathrm{~lx}$ at $4 \mathrm{~cm})$. One lamp was used to illuminate the drawing of the braille array for visual matches; the other two lights (behind the Masonite baffle) allowed the experimenter sufficient sight to keep track of the subjects' exploratory behavior. Observers were able to see the drawing of the braille code (the characters B-J) and letter equivalents as they touched the (invisible) tangible stimuli in the examination box. This allowed them to verbally name the letters that corresponded to the braille patterns that they touched.

Sight of finger angle was provided via two small LEDs, one placed on the upper surface (hairy skin) of the index finger (middle phalanx), and the other on the top of the fingernail of the exploratory fingertip (red LEDS; power dissipation of $60 \mathrm{~mW}$; luminous intensity of $6.3 \mathrm{mcd}$; each powered by a single $1.5-\mathrm{V}$ battery). Individuals observed tactual exploration through "stained glass." In the present experiment, the moderately dark-adapted observers could not see the array, the thumbtacks, or their fingers in relation to the background. The subjects were limited to sight of scanning movements (via the two LEDs) and could presumably generate visual representations of these movement patterns (see Heller, 1985). In addition, LEDs were used to provide a blurry line of light above the lines of braille in another condition, and a second pair of LEDs allowed the subjects vision of the angle of the finger.

Design and Procedure. The experiment was an independent group, one-way analysis of variance. The factor was the presence or absence of VG and the nature of visual information provided, with all tangible symbols tilted at $+90^{\circ}$ from the upright. A stopwatch was used to time the subjects on each line of braille. The subjects were given verbal guidance if they strayed from lines of braille or lost their place, but no other feedback was given. Prior to the experiment, the observers were shown a familiarization card with the embossed braille code that represented the letters A-J.

Exploration was limited to the tip of the index finger of the writing hand. All "reading" was from left to right, and the VG subjects were told to observe the LEDS on their fingers as they touched the braille. The subjects were told that the tangible patterns were tilted $+90^{\circ}$. In addition, a line drawing representing the orientation of the braille was present throughout, as was a drawing of the braille code with its letter equivalents. The observers made visual matches to the tangible code and verbally named the patterns they touched. 
In one control condition, the subjects were tested in the dark, with a small light above the braille code. The top of the braille box was covered with an opaque material for the groups that were not allowed visual guidance. In another group, the subjects looked through stained glass as they touched the braille, and viewed two LEDs on the top of the preferred, exploratory index finger; the second LED was placed between the two middle index finger joints (medial phalanx). This allowed vision of finger angle as these subjects explored the braille (2 LED-VG). A further group of subjects had two LEDS on their fingers, but also had a line of five LEDs above each line of braille. This gave the subjects visual frame of reference information and provided sight of the finger's angle in relation to vision of the angle of the lines of braille. It should be noted that the line of five LEDs appeared as one long, blurry red line when viewed through the stained-glass cover of the box. In both LED-VG conditions, the subjects were unable to see either their fingers or the surface they touched. An additional group of subjects touched the braille in normal room lighting (168 lx at the table surface), as in past research on orientation (Heller, 1987, 1992a).

\section{Results and Discussion}

The results show a large effect of the nature of visual guidance (see Table 1). A simple analysis of variance on the number correct showed a significant effect of visual information $[F(3,44)=4.3, p<.01]$. A Newman-Keuls test on mean number correct indicated that the dark control was not significantly different from the spatialreference VG condition $(p>.05)$, but both were superior to the normal light control mean and the 2 LED-VG group $(p<.05)$. The normal light group mean and the 2 LED-VG mean were not significantly different $(p>$ $.05)$. The effect of VG on mean time scores failed to reach significance $[F(3,44)=2.3, p=.09]$.

Providing visual information can either help or hurt haptics, depending on the nature and quality of the information. Performance with normal room lighting was like that in prior research (Heller, 1989b, 1992a), and comparable to that in the 2 LED-VG condition. This means that providing visual information on finger angle is not sufficient to aid tactile braille recognition. The superior performance of the group given information about finger angle and the angle of the line of braille implicates the importance of spatial reference information in tactual perception (see Millar, 1991).
What do we make of the superior performance of the group in low lighting? The low-light group did as well as the spatial-reference VG group. One possibility, of course, is that low lighting simply helps subjects attend to tactile input. Another hypothesis is that low light helps subjects engage in mental rotation of visual images as they feel the braille. This assumes that irrelevant visual information in the experimental environment may compete with resources required for mental manipulation of visual imagery of tactile input. The second experiment was designed to decide between these alternative explanations of the effect of low lighting.

It should be remarked that this analysis does not mean that VG has no effect beyond the attentional consequences of low lighting. It aided subjects with normal lighting, in pattern identification of tilted and upright braille. In addition, VG helped subjects avoid "getting lost" while scanning lines of braille (Heller, 1985, 1989b). It is important to note that the VG effect was obtained for both tilted and upright braille under conditions of normal room lighting (Heller, 1989b). It should be remarked that in prior demonstrations of the benefits of VG, subjects were able to see both the angles of their exploratory fingers and blue lines underneath the lines of braille.

\section{EXPERIMENT 2 \\ Low Versus Normal Room Lighting and Braille Words}

Two groups of subjects were exposed to braille words in normal lighting or low lighting. If the effect of low lighting was simply attentional, reduced illumination should help subjects read two-letter braille words.

\begin{abstract}
Method
Subjects. The subjects were 24 experimentally naive, sighted individuals recruited from the same undergraduate population. Eight blind subjects ( 5 congenitally blind and 3 late blind) were recruited at Industries of the Blind Workshops in Winston-Salem and Greensboro. These subjects identified themselves as fair or good braille readers. Blind volunteers were recruited to obtain an idea of "normal" "performance for reading braille words.

Stimuli and Apparatus. The stimuli were 25 two-letter words em-
\end{abstract} bossed in Grade 1 braille, taken from Heller and Mitchell (1985). A

Table 1

Mean Number Correct, Percent Correct, and Time (in Seconds) Per Character for $+90^{\circ}$ Tilted Braille (B-J) as a Function of Visual Guidance (VG) and Lighting

\begin{tabular}{lccccrr}
\hline \multicolumn{1}{c}{ Condition } & \multicolumn{2}{c}{ No. Correct } & & \multicolumn{2}{c}{ Time } \\
\cline { 2 - 6 } & $M$ & $S D$ & \% Correct & $M$ & $S D$ \\
\hline Dark & 18.1 & 5.8 & 67.0 & 12.5 & 5.5 \\
Control, normal lighting & 12.7 & 6.8 & 47.0 & 12.1 & 3.6 \\
VG (2 LEDs on finger) & 12.8 & 4.5 & 47.4 & 16.7 & 9.5 \\
VG (2 LEDs on finger and line of LEDs) & 18.6 & 4.1 & 68.9 & 19.2 & 10.5 \\
Normal lighting and VG (Heller, 1986b) & 17.9 & 5.1 & 66.3 & 19.5 & 7.3 \\
Blind subjects (Heller, 1992a) & 20.3 & & 75.2 & & \\
\hline
\end{tabular}

Note-Maximum score possible $=27 . S D$, standard deviation 
life-sized visual matching code illustrated the braille dot patterns and their letter equivalents. Normal spacing was used. The standard braille was embossed on plastic braille cards with a Perkins brailler, with about $1.4 \mathrm{~cm}$ of horizontal spacing between words. The words were arranged in six horizontal lines with four words in each line; a final line had one word. The beginning and end of each line were marked by the heads of tacks. The words were: la, at, by, do, up, to, ma, lo, is, hi, ho, pa, on, we, so, my, it, me, in, no, ha, go, be, am, and of.

The braille stimuli were placed flat on a horizontal shelf behind a large vertical panel. The front of the panel was covered with an opaque black cloth. The subjects could reach beneath the cloth to examine the braille. The only room lighting in the low-light condition consisted of the three submini lamps (.81 lx at $4 \mathrm{~cm}$ ) used in the low-light conditions of the first experiment. Normal room lighting was provided by overhead fluorescent ceiling fixtures, and measured $183 \mathrm{~lx}$ at the surface of the table

Procedure. The subjects read the two-letter words from left to right, and they were instructed to read each word and vocally identify the component letters. They used the tip of the index finger of the preferred (writing) hand. The subjects were timed with a stopwatch on each line of braille words. No time limits were imposed, and feedback was not given.

\section{Results and Discussion}

Lighting had minimal effect on reading braille words (see Table 2), and the effect on number of words correct was nonsignificant $[t(22)=1.2, p>.05]$. Lighting had no impact on the number of letters correct $(t=1)$, or on mean time scores $[t(22)=1.2, p>.05]$. It was not surprising that blind subjects were much faster and far more accurate than the sighted subjects. Much of the "reading time" of the blind subjects was taken up by the time required to read the words and component letters aloud.

The results of this experiment are not consistent with a simple attentional interpretation of the effect of low lighting on braille recognition. Low lighting did not significantly improve the reading of upright braille in the present experiment. This means that the benefits of low lighting are probably specific to tasks that require mental manipulation of imagery, as with $+90^{\circ}$ rotated braille. Braille recognition in the congenitally blind does not depend on rotation of visual images, yet the congenitally blind are able to compensate for $+90^{\circ}$ rotated braille (Heller, 1992a). The sighted, however, are likely to engage in visualization (see Revesz, 1950). It is likely that low lighting helps the sighted by reducing distractions from extraneous visual input. The presence of irrelevant visual input is likely to present a problem only when the subjects' mental resources are engaged in the manipulation of visual imagery. This is the case with rotated braille.

Table 2

Mean Number of Words Correct, Letters Correct, and Time per Word (in Seconds)

\begin{tabular}{lcccccc}
\hline & \multicolumn{2}{c}{$\begin{array}{c}\text { Words } \\
\text { Correct }\end{array}$} & & \multicolumn{2}{c}{$\begin{array}{c}\text { Letters } \\
\text { Correct }\end{array}$} & Time \\
\cline { 2 - 3 } \cline { 5 - 7 } & $M$ & $S D$ & & $M$ & $S D$ & per Word \\
\hline Normal lighting & 7.1 & 4.6 & & 22.5 & 9.2 & 49.1 \\
Low lighting & 10.0 & 7.5 & & 27.1 & 12.8 & 57.9 \\
Blind subjects $(n=8)$ & 25.0 & 0 & & 50.0 & 0 & 1.7 \\
\hline
\end{tabular}

Note-Maximum possible number of words correct $=25 . S D$, standard deviation.
How, then, can we explain the beneficial effects on both the spatial-reference VG group and the control group without VG but in relative darkness? One might argue that the high performance of the control group with low lighting means that it is not necessary to provide spatial reference information for touch. Given this interpretation, one might explain away the spatial-reference VG effects in terms of low lighting. However, the present results do not mean that normal VG effects can be explained solely by low lighting. VG can aid haptic pattern recognition with normal lighting and upright tangible patterns (Heller, $1989 \mathrm{~b}$ ). The benefits of VG are clearly tied to adequate spatial reference information.

It is possible that the distracting effects of normal room lighting extend to the ability of subjects to make use of spatial reference information. That is, irrelevant visual stimulation in an experimental environment may impede the ability of subjects to make use of substitute visual reference cues. The visual background provided by dark, blurry sight of the stimulus exposure box and the vertical baffle might provide spatial references within which to localize tactile stimulation. In normal room lighting, however, extraneous visual input may draw focal attention and impede haptics. We also cannot rule out the possibility that more than one mechanism is available to aid haptics. Given this alternative interpretation, we might improve haptic form perception in a number of different ways, including improved imagery and/or added spatial reference information. These mechanisms are not mutually exclusive.

\section{GENERAL DISCUSSION}

There was a substantial improvement in haptic pattern identification when VG of touch was provided. The results of the first experiment showed that VG may aid or hinder tactile perception, depending on the quality of information that is given. The subjects required more than visual information about finger angle alone, since the 2 LED-VG group showed much lower performance than did subjects given sight of both finger angle and the angle of the lines of braille. This implicates the importance of spatial reference information for touch. Furthermore, low lighting helped subjects cope with $+90^{\circ}$ tilted braille in the first experiment, but had no influence on reading upright, two-letter braille words in Experiment 2. This means that low lighting aids pattern perception by reducing the distracting effects of irrelevant visual input, when subjects need to engage in the mental rotation of visual imagery. ${ }^{1}$ The results were consistent with the idea that sighted subjects often engage in visual imagery when touching patterns and making visual matches (see Klatzky, Lederman, \& Matula, 1991; Revesz, 1950).

Sighted subjects tend to show rather poor performance when confronted by $+90^{\circ}$ tilted braille, but VG can improve recognition to levels that are close to that of blind readers of braille (see Heller, 1992a). These results sug- 
gest that part of the low performance we may see in tactile pattern recognition tasks comes from the loss of this sort of spatial reference information. Visual information about the relationship between one's limbs and the orientation of tangible arrays optimizes performance. VG of touch can aid pattern perception by improving the precision of exploratory movements.

The beneficial effects of low lighting provide support for the idea that sighted subjects frequently engage in visualization when touching patterns that they cannot see. Late blind subjects also describe thinking of space in terms of visual images (see Heller, 1989a; Hollins, 1989). The effect of low lighting was dependent on the mental rotation task of the first experiment, suggesting that extraneous visual stimulation, under normal room lighting, may interfere with the process of generating and manipulating visual images. Thus, subjects in a variety of pattern perception tasks were observed closing their eyes in an attempt to visualize patterns. This effect is not just a consequence of improved attention, since it did not generalize to the recognition of upright, two-letter words.

In normally sighted people, VG is typically the function of peripheral vision, whereas foveal vision may engage in pattern perception and recognition of objects. Thus, vision and touch can often operate in a cooperative manner, with vision used for guidance of the hand to optimize haptic perception (see Heller, 1982). Other forms of cooperation may take the form of touch's reorienting objects to facilitate viewing (Ruff, 1989). Foveal vision operates much faster than touch in pattern perception, and sighted people may be more likely to rely on vision for judgments of form or shape when a discrepancy is presented to the senses (see Rock \& Victor, 1964). Peripheral vision, however, may not be especially good for pattern perception tasks, since it is blurry. Heller (1983) found that blurry vision (via stained glass) yielded haptic dominance in a shape recognition task. More recently, Heller (1992b) reported haptic dominance in a form perception task when vision was used to guide haptic exploration. In that experiment, vision was limited to a mirror reflection that produced top-bottom reversals. The results of these experiments, taken together, suggest that it is important to distinguish between the functions of vision, namely those of pattern perception or guidance.

Turvey (1977, p. 254) has discussed physiological evidence for distinguishing between the visual functions of identification of objects or things and the specification of complex spatial relationships and localization (also see Hester, 1977). Neurological patients may show normal locomotion-related activities of the arms and hands, with proper coordination. However, the two hands may not function cooperatively in the same patients when focal vision is involved. It should be noted that the visual function of identification of an object can be distinguished from perception of object characteristics (Gibson, 1979). One may perceive an object accurately, yet not know how to name it (Dretske, 1990).

The visual imagery explanation of the present results is not inconsistent with the obtained VG effects. It is prob- able that these two functions are complementary routes toward intersensory facilitation. Both mechanisms depend on the shift of visual functioning away from focal, or foveal, vision toward the guidance function - that is, peripheral vision. Furthermore, subjects may make use of "higher" level functions when engaged in haptic shape matching or pattern naming. According to this view, subjects will use any available information or aid, including spatial reference information or visual imagery. Experimental procedures may often selectively interfere with the ability of subjects to use spatial reference information (e.g., visual occlusion of the hand). Normal room lighting could prevent subjects from effectively visualizing the patterns they touch. The data do not force the idea that visual imagery is necessary for haptics. Clearly, the mental rotation of tactually derived patterns does not depend on visual imagery in the congenitally blind (Heller, 1992a).

The results of these experiments are relevant to a current theoretical fomulation in haptics. Klatzky, Lederman, and their colleagues have repeatedly proposed that touch is better suited for the perception of substance-related characteristics of objects, such as texture (see Heller, 1989c), hardness, thermal attributes, and so forth (see, e.g., Lederman \& Klatzky, 1987). Moreover, they propose that vision is more appropriate for the perception of twodimensional displays, where touch is poor (Lederman, Klatzky, Chataway, \& Summers, 1990). The assumption is that touch fails at two-dimensional pattern perception because of such factors as memory load and low spatial resolution for touch. Lederman and Klatzky have suggested that visual imagery may aid haptics when tasks involve two-dimensional configurations. The present results are consistent with some aspects of this theoretical view, since they suggest that foveal visual information can disrupt tactile pattern perception. A variety of tasks may promote visualization, and it is in these tasks that foveal vision may interfere with haptics. Thus, it is likely that the subjects in a typical intersensory conflict paradigm rely on foveal vision for their judgments and never really use touch to discover the nature of the forms that have been presented. It may be rather difficult to notice certain forms with the sense of touch while looking at them with normal foveal vision. We typically rely on sight for pattern perception, since it is faster and more efficient than touch. It may not be easy for sighted subjects to feel a shape and generate a visual image derived from touch while simultaneously directing foveal visual attention to a configuration. Moreover, circumstances that promote or mimic the guidance function of vision may lead to haptic dominance (Heller, 1983, 1992b). Again, haptic dominance was found in a letter-naming task when vision was used to guide the finger to embossed patterns.

The foregoing argument is clearly limited to normally sighted observers with clear foveal vision. Under other less optimal circumstances, intersensory attentional habits may change. Thus, sighted subjects are rather poor at some haptic pattern perception tasks. It is likely that much of their lower performance reflects a lack of education (Heller, 1989a). Certainly, blind subjects are much faster at read- 
ing braille, and far less likely to make errors (see Table 1; Heller, 1992a). It is extremely rare to find errorless performance in sighted subjects engaged in tactile pattern perception. Consequently, low tactile performance in pattern recognition tasks may be a result of a lack of education of the sense of touch, and this lower level of skill could, in turn, affect reliance on the senses (see Heller, 1989a, 1989b). Moreover, in tasks where touch is better than vision, such as detection of the smoothest textures, subjects may prefer touch (see Heller, 1982, 1989c).

The present results have considerable application to issues involving the rehabilitation of visually impaired people. However, simplistic recommendations are not possible. There are occasions when it is appropriate to encourage the use of remaining residual vision for guidance of touch. Alternatively, there may be other circumstances where this may not be a prudent strategy. People with low vision have often been blindfolded, in an attempt to optimize touch and instruction in mobility skills (see, e.g., Lauren, 1988). The present results suggest that these educators may not always have been off the mark. Visual information can sometimes impede attention to tactual pattern information in tasks that may require mental rotation, such as map reading and mobility. It is especially important to be sure that when visual information is made available, it should transmit spatial reference information. The presence of some sorts of visual input can attract foveal visual attention and disrupt the processing of tactual pattern information. This is the case for the provision of a single LED or two LEDs on the exploratory finger. Even irrelevant stimulation that is observed under normal room lighting can disrupt attention to tactual information in mental rotation tasks. These results are also consistent with a recent report by Rieser, Hill, Talor, Bradfield, and Rosen (1992) on the importance of visual experience for the development of mobility in visually impaired individuals. In their study, broad-field visual experience seemed more important for the development of mobility skills than did visual acuity. Thus, subjects with early deficits in broad-field vision showed lower performance than did visually impaired subjects with early visual acuity losses. Rieser et al.'s results are consistent with the idea that vision may serve different functions-namely, that of localizing stimuli and guidance of locomotion, or that of pattern identification.

\section{REFERENCES}

Attneave, F., Benson, B. (1969). Spatial coding of tactual stimulation. Journal of Experimental Psychology, 81, 216-222.

DreTSKe, F. (1990). Seeing, believing, and knowing. In D. N. Osherson, S. M. Kosslyn, \& J. M. Hollerbach (Eds.), Visual cognition and action (Vol. 2, pp. 129-148). Cambridge, MA: MIT Press.

FoulKe, E. (1991). Braille. In M. A. Heller \& W. Schiff (Eds.), The psychology of touch (pp. 219-233). Hillsdale, NJ: Erlbaum.

GiBson, J. J. (1979). The ecological approach to visual perception. Boston: Houghton Mifflin.
Heller, M. A. (1982). Visual and tactual texture perception: Intersensory cooperation. Perception \& Psychophysics, 31, 339-344.

Heller, M. A. (1983). Haptic dominance in form perception with blurred vision. Perception, 12, 607-613.

HELle R, M. A. (1985). Tactual perception of embossed Morse code and braille: The alliance of vision and touch. Perception, 14, 563-570.

HeLLER, M. A. (1987). The effect of orientation on visual and tactual braille recognition. Perception, 16, 291-298.

Heller, M. A. (1989a). Picture and pattern perception in the sighted and blind: The advantage of the late blind. Perception, 18, 379-389.

Heller, M. A. (1989b). Tactile memory in sighted and blind observers: The influence of orientation and rate of presentation. Perception, 18, 121-133.

Heller, M. A. (1989c). Texture perception in sighted and blind observers. Perception \& Psychophysics, 45, 49-54.

Heller, M. A. (1992a). The effect of orientation on tactual braille recognition: Optimal "touching positions." Perception \& Psychophysics, 51, 549-556

Heller, M. A. (1992b). Haptic dominance in form perception: Vision versus proprioception. Perception, 21, 655-660.

Heller, M. A., \& Mitchell, B. Y. (1985). Helping the new braille reader: The effects of spacing, finger locus, and gloves. Perceptual \& Motor Skills, 61, 363-369.

Hester, M. (1977). Visual attention and sensibility. In R. Shaw and J. Bransford (Eds.), Perceiving, acting, and knowing (pp. 135-169). Hillsdale, NJ: Erlbaum.

Holuns, M. (1989). Understanding blindness. Hillsdale, NJ: Erlbaum. Klatzky, R. L., Lederman, S. J., \& Matula, D. E. (1991). Imagined haptic exploration in judgments of object properties. Journal of Experimental Psychology: Learning, Memory, \& Cognition, 17, 314-322.

LAUREN, H. (1988). To blindfold or not to blindfold? Is that the question for O\&M instructors? Journal of Visual Impairment \& Blindness, $82,150$.

LedERMAN, S. J., \& KLATZKY, R. L. (1987). Hand movements: A window into haptic object recognition. Cognitive Psychology, 19, 342-368.

Lederman, S. J., Klatzky, R. L., Chataway, C., \& Summers, C. D. (1990). Visual mediation and the haptic recognition of twodimensional pictures of common objects. Perception \& Psychophysics, 47, 54-64.

McKinney, J. P. (1964). Hand schema in children. Psychonomic Science, 1, 99-100.

MiLlaR, S. (1981). Cross-modal and intersensory perception and the blind. In R. D. Walk \& H. L. Pick, Jr. (Eds.), Intersensory perception and sensory integration (pp. 281-314). New York: Plenum.

Millar, S. (1991). A reverse lag in the recognition and production of tactual drawings: Theoretical implications for haptic coding. In $\mathbf{M}$. A. Heller \& W. Schiff (Eds.), The psychology of touch (pp. 301-325). Hillsdale, NJ: Erlbaum.

Pick, H. L., JR. (1974). The visual coding of non-visual spatial information. In R. B. MacLeod \& H. L. Pick, Jr. (Eds.), Perception: Essays in honor of James J. Gibson (pp. 153-165). Ithaca, NY: Cornell University Press.

REvesz, G. (1950). The psychology and art of the blind. London: Longmans Green.

Rieser, J. J., Hill, E. W., Talor, C. R., Bradfield, A., \& Rosen, S. (1992). Visual experience, visual field size, and the development of nonvisual sensitivity to the spatial structure of outdoor neighborhoods explored by walking. Joumal of Experimental Psychology: General, 121, 210-221.

Rock, I., \& VICTOR, J. (1964). Vision and touch: An experimentally created conflict between two senses. Science, 143, 594-596.

RuFF, H. A. (1989). The infant's use of visual and haptic information in the perception and recognition of objects. Canadian Joumal of Psychology, 43, 302-319.

TuRveY, M. T. (1977). Preliminaries to a theory of action with reference to vision. In R. Shaw \& J. Bransford (Eds.), Perceiving, acting, and knowing (pp. 211-265). Hillsdale, NJ: Erlbaum. 
WARREN, D. H., \& Rossano, M. J. (1991). Intermodality relations: Vision and touch. In M. A. Heller \& W. Schiff (Eds.), The psychology of touch (pp. 119-137). Hillsdale, NJ: Erlbaum.

\section{NOTE}

1. The low-lighting effect was also found with $+180^{\circ}$ rotated standard braille. Performance was significantly better with $+180^{\circ}$ braille in the dark ( $M=14.6)$ than it was with normal lighting ( $M=11.5$ ) $[t(22)=2.4, p<.05]$. Low lighting had no effect on upright, jumbo braille $[\mathrm{b}-\mathrm{t}$, maximum score possible $=36 ; M$ (light $)=27.5, M$ (dark) $=$ 27.7].

(Manuscript received September 8, 1992: revision accepted for publication April 29, 1993.) 